\title{
Presenza della glutatione perossidasi plasmatica (pIGPX) in pazienti affetti da epatite C
}

\author{
Angela Giannattasio, Chiara Coppola, Giovanna Panza, Erasmo Falco, Giuliana Di Prisco, \\ Giuseppe Scancariello, Annarita Cimmino', Letizia Spadaro, Riccardo Smeraglia \\ Virologia P.O. “C. Ascalesi” - ASL NAI \\ 'Laboratorio di Patologia Clinica
}

Presence of plasmatic glutathione peroxidase (pIGPX) in patients affected by HCV

Key Words: HCV, pIGPX, C hepatitis

\section{SUMMARY}

In the past decade it became accepted that reactive oxygen metabolites (ROM's) play a role in various tissues damages, thus in certain liver deseases as well hepatitis C. Hepatic cells, utilize the enzymatic system constituted by the glutathione peroxidase/glutathione reductase enzymes (GPX/GR), to reduce oxidative damages. In fact, the GPX enzyme utilizes GSH to reduce ROM's. Interferon alpha (IFN) might be useful to reducing the oxidative stress, probably through an increase of reduced glutatione (GSH). Recent findings demonstrate that hepatitis $\mathrm{C}$ virus $(\mathrm{HCV})$ encodes a GPX gene in a reading frame overlapping a known non structural gene, NS4b, of unidentified function. The HCV GPX sequence has features of both the plasma and cellular GPX types.

Aim of our study was to demonstrate the presence of the PIGPX in sera of patients with C hepatitis and with a various viremia level. Our results show the presence of pIGPX in all the sera examinated. Particularly, there is a remarkable increase in the sera with an high viremia as respect the sera without a viral replication. Moreover, it is very interesting to observe that some of the sera without viral replication possess an high pIGPX level. In the other side, some of the sera with an high viral replication have low pIGPX levels. Starting from these results, it will be foundamental to evaluate whether the patients examinated are, or not, in terapeutic treatment. Moreover, since GPX is a target of the tumor suppressor protein p53, that activates the apoptotic process in mammalian cells, it will be very interesting to establish wether the pIGPX detected in the sera is of viral source or is the pIGPX present in the hepatocytes. In fact, the understandig of these cellular mechanisms might play an important role in the evolution of hepatic cirrosis to hepato carcinoma in patients with $\mathrm{C}$ hepatitis.

\section{INTRODUZIONE}

L'infezione da virus epatitico $\mathrm{C}(\mathrm{HCV})$ costituisce uno dei maggiori problemi sanitari su scala mondiale. L'epatite $\mathrm{C}$ è una malattia subdola, paucisintomatica, o del tutto asintomatica, caratterizzata da un'alta percentuale $(85 \%)$ di evoluzione verso la cronicizzazione.

Il $10-20 \%$ circa dei pazienti affetti da epatite C cronica evolve verso la cirrosi epatica, in un arco di tempo medio di 1-3 decadi. Ogni anno, il 5\% circa di questi casi subisce un'ulteriore evoluzione verso il carcinoma primitivo del fegato $(1,3)$. Nei soggetti affetti da epatite $\mathrm{C}$ è stato riscontrato un aumento dei metaboliti reattivi dell'ossigeno (ROM's). L'aumento dello stress ossidativo, negli epatociti, giocherebbe un ruolo chiave nell'evoluzione delle epatopatopie da virus $\mathrm{C}$ in cancrocirrosi (9); il trattamento con $\alpha$ interferone porterebbe ad una diminuzione dei ROM's e, quindi, dello stress ossidativo in queste cellule (8). Il meccanismo di azione dell' $\alpha$ interferone è, in parte, ancora oggetto di studio anche se dati recenti dimo- strano un effetto di questa molecola sul metabolismo del glutatione e sui meccanismi alla base della regolazione del ciclo cellulare $(7,8)$.

Uno dei più importanti sistemi anti-ossidanti, presenti nelle cellule umane, comprese quelle epatiche, è quello costituito dagli enzimi Glutatione Perossidasi/Glutatione Reduttasi (GPX/GR). L'enzima GPX utilizza il Glutatione ridotto (GSH) per neutralizzare l'aumento dei ROM's nelle cellule.

Al momento, sono state isolate le sequenze di 5 GPX: a) GPX1 o forma citoplasmatica; b) GPX2 o forma gastrointestinale; c) GPX3 o plGPX o forma plasmatica; d) GPX4 o fosfolipide idroperossido-Glutatione Perossidasi di membrana; e) GPX5 o non selenio-GPX o forma epididimale (2).

Studi recenti, che utilizzano metodi hanno dimostrato che l'HCV codifica per una sequenza omologa alla GPX, in particolare alla plGPX e alla GPX1 (14). La regione genomica dell'HCV, che presenta questa notevole omologia di sequenza 
con le suddette GPX, è situata nella giunzione dei geni non strutturali NS4b e NS4a, la cui funzione è in parte ancora sconosciuta (15).

Partendo dai dati presenti in letteratura, lo scopo del nostro studio è stato quello di dimostrare la presenza della proteina enzimatica plGPX nel siero di pazienti affetti da epatite $\mathrm{C}$, con vario grado di viremia. La plGPX, infatti, soprattutto se di origine virale, potrebbe avere un ruolo fondamentale nella replicazione dell'HCV e, di conseguenza, nella progressione della malattia verso il carcinoma primitivo del fegato.

\section{MATERIALI E METODI}

Sono stati esaminati i sieri di 35 pazienti positivi per gli anticorpi anti HCV (HCV-Ab). Di questi pazienti, 12 risultavano in fase non replicativa, 9 con bassa viremia, e 14 con alta viremia (Cobas Amplicor HCV Monitor Ver.2 Roche Diagnostics S.p.A.).

Per dimostrare la presenza dell'enzima GPX è stato utilizzato un metodo immunoenzimatico (ELISA) specifico per la plGPX umana (BIOX$\mathrm{TECH}^{\circledR} \mathrm{pl} \cdot \mathrm{GPX}$ Enzyme Immunoassay ${ }^{\mathrm{TM}}$ ). La presenza della proteina enzimatica plGPX è rivelata da un anticorpo policlonale biotinilato, diretto verso l'enzima. Il passaggio finale del metodo prevede un'amplificazione biotina-streptavidina, in cui la streptavidina è legata covalentemente alla fosfatasi alcalina.

La plGPX viene quantificata nel siero mediante l'idrolisi enzimatica del p-nitrofenil fosfato (pNPP) e la rivelazione avviene su micropiastra, con lettura a $405 \mathrm{~nm}$. Il metodo, inoltre, prevede la messa a punto di una curva standard, costruita partendo da $4.2 \mathrm{ng} / \mathrm{mL}$ fino ad un massimo di 300 $\mathrm{ng} / \mathrm{mL}$ di proteina. La curva raggiunge il plateau dopo $20 \mathrm{~min}$, a $37^{\circ} \mathrm{C}$ (figura I). I risultati dei sieri in esame vengono estrapolati partendo dalla curva standard.

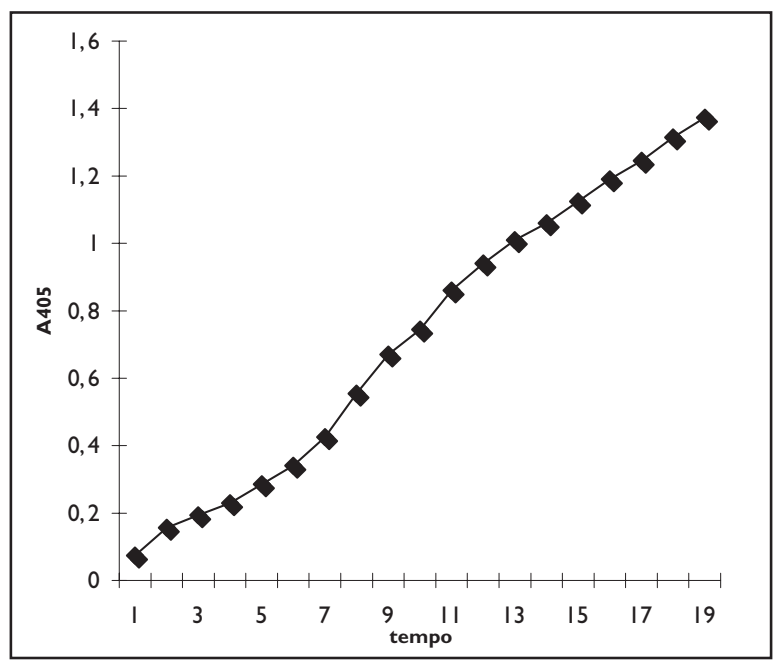

Figura I. Curva standard della plGPX

\section{RISULTATI}

I risultati da noi ottenuti dimostrano che nei sieri dei pazienti con alta viremia (N. 10 sieri) si rileva un contenuto di plGPX decisamente più alto rispetto ai sieri dei pazienti in fase non replicativa (N. 9 sieri), mentre nei sieri dei pazienti con bassa viremia (N. 9 sieri) si rileva un valore di plGPX intermedio rispetto ai 2 gruppi precedenti. É estremamente interessante notare come nel gruppo dei pazienti in fase non replicativa si rilevano 3 sieri con un elevato valore di plGPX e, viceversa, nel gruppo di pazienti con alta viremia si rilevano 4 sieri che presentano un basso valore di plGPX (tabella 1).

Tabella I. Quantità di pIGPX ( $\mu \mathrm{g} / \mathrm{mL})$ presente nei sieri dei pazienti HCV-Ab positivi

\begin{tabular}{lccccc}
\hline & FASE NON & FASE NON & BASSA & ALTA & ALTA \\
& REPLICATIVA & REPLICATIVA & VIREMIA & VIREMIA & VIREMIA \\
\hline N. SIERI & & & & & \\
ESAMINATI & 9 & 3 & 9 & 10 & 4 \\
\hline GPX3 $(\mu \mathrm{g} / \mathrm{mL})$ & $6.33 \pm 0.9$ & $11.98 \pm 0.7$ & $9.84 \pm 0.8$ & $12.40 \pm 0.7$ & $6.95 \pm 0.6$ \\
\hline
\end{tabular}

\section{CONCLUSIONI}

É ormai noto che i ROM's, la perossidazione dei fosfolipidi di membrana e i sistemi di difesa antiossidanti (GPX/GR), rientrano nell'etiopatogenesi delle epatopatie da virus C. Infatti, nei soggetti affetti da epatite $\mathrm{C}$, è stata dimostrata un'alterazione della bilancia ossidanti/antiossidanti, che determina un aumento dello stress ossidativo. Studi recenti dimostrano che l'HCV codifica per una GPX, che mostra una notevole omologia di sequenza con le sequenze di diverse GPX già note in letteratura (15). I risultati del nostro lavoro dimostrano che la proteina plGPX è presente nei sieri di tutti i pazienti arruolati per il nostro studio. In particolare, si rileva un netto aumento della plGPX nei sieri dei pazienti in fase di alta replicazione virale rispetto ai sieri dei pazienti in fase non replicativa. Inoltre, fra i sieri in fase non replicativa, alcuni mostrano un valore di plGPX paragonabile a quello riscontrato nei sieri in fase di alta viremia. Viceversa, alcuni sieri in fase di alta viremia mostrano una quantità di plGPX simile a quelli in fase non replicativa.

Alla luce dei dati ottenuti si aprono affascinanti interrogativi sia sul ruolo della plGPX nelle epatopatie da virus $\mathrm{C}$ e sia sull'origine della proteina da noi dosata (virale o umana).

A tutt'oggi, non sono ancora completamente noti i meccanismi molecolari alla base dell'effetto di trasformazione neoplastica nei pazienti affetti da cirrosi da virus $\mathrm{C}$. Il riscontro di quantità di virus $\mathrm{C}$ significativamente più elevate nei tumori più differenziati rispetto ai tipi istologici scarsamente differenziati, suggerisce che le cellule epatiche trasformate diventino progressivamente meno 
permissive alla replica dell'HCV. Inoltre, è sempre più evidente l'associazione tra infezione da genotipo $1 \mathrm{~b}$ e forme più avanzate di epatopatia ed epatocarcinoma $(5,6)$. La proteina virale non strutturale NS3 è in grado di modulare l'espressione di diversi geni della cellula ospite e di interferire con i processi apoptotici cellulari. Ancora, la proteina non strutturale NS5a svolge un ruolo chiave nella replicazione dell'HCV ed è implicata nei meccanismi di resistenza alla terapia con interferone $(10,11)$. Recenti studi hanno dimostrato che NS5 è in grado di reprimere la trascrizione del gene $\mathrm{p} 21$ coinvolto nella regolazione del ciclo cellulare (4).

L'apoptosi o morte cellulare programmata è un processo che diminuisce, o si blocca del tutto, nelle patologie autoimmuni e nel cancro. La proteina p53 attiva il processo apoptotico attraverso dei target cellulari. Oltre alla già nota proteina p21, studi recenti hanno dimostrato che un nuovo target della p53 è la GPX $(12,13)$.

Alla luce degli ultimi dati presenti in letteratura sull'argomento, e partendo dai risultati del nostro studio, è estremamente importante non solo l'ampliamento della nostra casistica, ma anche il monitoraggio dei pazienti stessi (epatopatia $\mathrm{C}$ in fase iniziale e/o soggetti in trattamento con IFN ) nonchè la genotipizzazione dell' $\mathrm{HCV}$.

Visto, inoltre, il ruolo che la GPX potrebbe avere nell'attivazione dei processi apoptotici cellulari, diventerà estremamente necessario stabilire se la plGPX rilevata nei sieri dei pazienti in esame sia di origine virale o provenga dalla cellula ospite. Quest'ultimo punto potrebbe essere di grande importanza per la comprensione dei meccanismi alla base dell'evoluzione dell'epatopatia da HCV verso il carcinoma primitivo del fegato.

\section{BIBLIOGRAFIA}

1. Alter MJ. Epidemiology of hepatitis C. Hepatology 1997; 26: 62S-5S.

2. Giannattasio A, Girotti M, Williams $\mathrm{K}$, Hall L, Bellastella A. Puberty influences expression of phospholipid hydroperoxide glutathione peroxidase (GPX4) in rat testis. Probable hypophysis regulation of the enzyme in male reproductive tract. J Endocrinol Invest 1997; 20: 439-44.

3. Kato N. Molecular virology of hepatitis C virus. Acta Med Okayama 2001; 55 (3): 133-59.

4. Keng-Hsin Lan, Meei-Ling Sheu, Shinn-Jang Hwang, et al. HCV NS5A interacts with p53 and inhibits p53-mediated apoptosis. Oncogene 2002; 21 : 4801-11.

5. Koike K, Tsutsumi T, Fujie H, et al. Molecular mechanism of viral carcinogenesis. Oncology 2002; 62(suppl 1): 29-37.

6. Koike K, Moriya K, Kimura S. Role of hepatitis C virus in the development of hepatocellular carcinoma: transgenic approach o viral hepatocarcinogenesis. J Gastroenterol Hepatol 2002; 17: 394-400.
7. Mutlu-Turkoglu U, Ademoglu E, Turkoglu S, Badur S, Uysal M, Toker G. The effects of interferon-alpha on serum lipid peroxidation and total thiol content in patients with chronic active hepatitis-C. Res Commun Mol Pathol Pharmacol 1997; 96 (3): 357 61.

8. Neri S, Ierna D, Antoci S, Campanile E, D'Amico RA, Noto R. Association of alpha-interferon and acetyl cysteine in patients with chronic $\mathrm{C}$ hepatitis. Panminerva Med 2000; 42 (3): 187-92.

9. Par A, Roth E, Rumi G Jr, Kovacs Z, Nemes J, Mozsik G. Oxidative stress and antioxidant defense in alcoholic liver disease and chronic hepatitis C. Orv Hetil 2000; 141 (30): 1655-9.

10. Pawlotsky JM, Germanidis G. The non-structural 5A protein of hepatitis C virus. J Viral Hepatitis 1999; 6: 343-56.

11. Ruster B, Zeuzem S, Krump-Konavalinkova V, et al. Comparative sequence analysis of the core and NS5region of hepatitis $\mathrm{C}$ virus from tumor and adjacent non-tumor tissue. J Med Virol 2001; 63: 128-34.

12. Sun Y. Identification and characterization of genes responsive to apoptosis: application of DNA chip technology and mRNA differential display. Histol Histopathol 2000; 15 (4): 1271-84.

13. Tan M, Li S, Swaroop M, Guan K, Oberley LW, Sun Y. Transcriptional activation of the human glutathione peroxidase promoter by p53. J Biol Chem 1999; 274 (17): 12061-6.

14. Zhang W, Ramanathan CS, Nadimpalli RG, Bhat AA, Cox AG, Taylor EW. Selenium-dependent glutathione peroxidase modules encoded by RNA viruses. Biol Trace Elem Res 1999; 70 (2): 97-116.

15. Zhang W, Cox AG, Taylor EW. Hepatitis C virus encodes a selenium-dependent glutathione peroxidase gene. Implications for oxidative stress as a risk factor in progression to hepatocellular carcinoma. Med Klin 1999; 94 Suppl 3: 2-6.

\section{Angela Giannattasio,}

Via Raffaele Carrascosa 10/D

80141 Napoli

Tel.: $0817516067 / 0812542124$ - 2127;

Fax: $0812542061-2123$

e-mail: angelagiannattasio@yahoo.it 